\title{
Build of Dust Removal System Controlled by DCS and PLC Two-level Supervisory Control System
}

\author{
Qi Wang, Zhumei Sun, Jianyun Bai, Tianxing Han and Shaohong Liu \\ Automation department of Shanxi University, Taiyuan 030013, China
}

\begin{abstract}
The dust removal system of a certain power plant was controlled by omron programmable Logic controller (PLC). It needs to be included in the master control system DCS (distributed control system) in order to fit the request of all control systems integration. Using a network protocol converter realize the Profibus-DP and DeviceNet fieldbus networks communication, which are two networks with heterogeneous protocol. So a two levels supervisory control system is built. The Siemens PCS7 is the main control system, and omron PLC is the control subsystem. By Siemens PCS7 system configuration, the upper level monitoring program CFC configuration, remote monitoring picture configuration, the gateway PD-100S Settings and omron PLC system configuration, the dust removal system is included in power plant main control system PCS7. The retrofit scheme needs small amount of new equipments. Hardware and software configuration and parameters setting are simplfy. Under the existing dedusting system data update quantity, the system can reliably realize the remote monitoring. The scheme can provide a reference for the integration of heterogeneous fieldbus system.
\end{abstract}

Keywords-protocol converter; fieldbus; distributed control system; heterogeneous compatible; remote monitoring; integration transformation

\section{INTRODUCTION}

The dust removal system of a power plant was controlled by an Omron Programmable Logic Controller (PLC). The auxiliary control system was not included in the power plant's main control DCS (Distributed Control System). In order to respond to the requirements of integration of the whole plant's management and control of modern power plants, the dust removal system needs to be integrated into the power plant's main control system. Since the fieldbus communication protocol of the slave control and master control system is incompatible, there are two alternative retrofit schemes. Solution 1: The existing dust removal system controlled by Omron PLC is abolished, and the master control device PCS7 is used to implement the control and the dust control system is included. Master PCS7 system. This solution requires repurchasing, installing and debugging remote $\mathrm{I} / \mathrm{O}$, and reconfiguring the PCS7 system. The new system has a long constructive cycle and high cost [1]. Solution 2: Base on the existing systems, build compatible systems for heterogeneous fieldbus protocol communications. This solution realize the information exchange between the auxiliary control Omron PLC system and the main control PCS7 by configuring the gateway. Which can not only expand the control range of the main control system, but also shorten the time from the design installation to the commissioning of the transformation project, as well reducing the investment in retrofit. Solution 2 is a preference plan, and a double-layer remote monitoring dust removal system compatible with DCS and PLC is constructed.

\section{The WORKING PRINCIPLE AND CONTROL OF DUST REMOVAL SYSTEM}

The dusty flue gas from the boiler first enters the dust removal intake smoke box and is evenly distributed through the second layer porous air distribution plate. After that, the dusty flue gas enters the dust chamber, and the dust is mostly filed on the outer surface of the cloth bag by the bag filter. The filtered flue gas passes through the bag and is discharged from the outlet of the net air chamber [2].

The dust collector is provided with two reciprocating moving sub-compartment-positioning and counter -blowing units. Each counter-blowing unit cleans the dust bag rooms in turn according to the differential pressure command. As the filtering time increases, the soot layer that is trapped on the outer surface of the bag is continuously thickened. When the resistance increases, the differential pressure signal measured by the differential pressure transmitter is higher than the set value, the counter-blow cleaning system is started. Opening the dust removal valve, starting the dust removal motor and the counter-blowing fan. The air is introduced, and after being heated by the heat exchanger, blown into the bag room which needs dust removal. The Automatic start flow chart of dust removal control is shown in Figure I.

When a counter-blow mechanism is working, the other counter-blow mechanism is in out off operating state, and the corresponding dust removal valve should be in a closed state.

When the dust-clearing makes the differential pressure signal measured by the differential pressure transmitter lower than the set value, dust-clearing work is stopped automatically, and then, the counter-blowing fan is stopped, the dust removal motor is stopped, and the dust removal valve is closed. The automatic stop flow chart of dust removal control is shown in Figure II. 


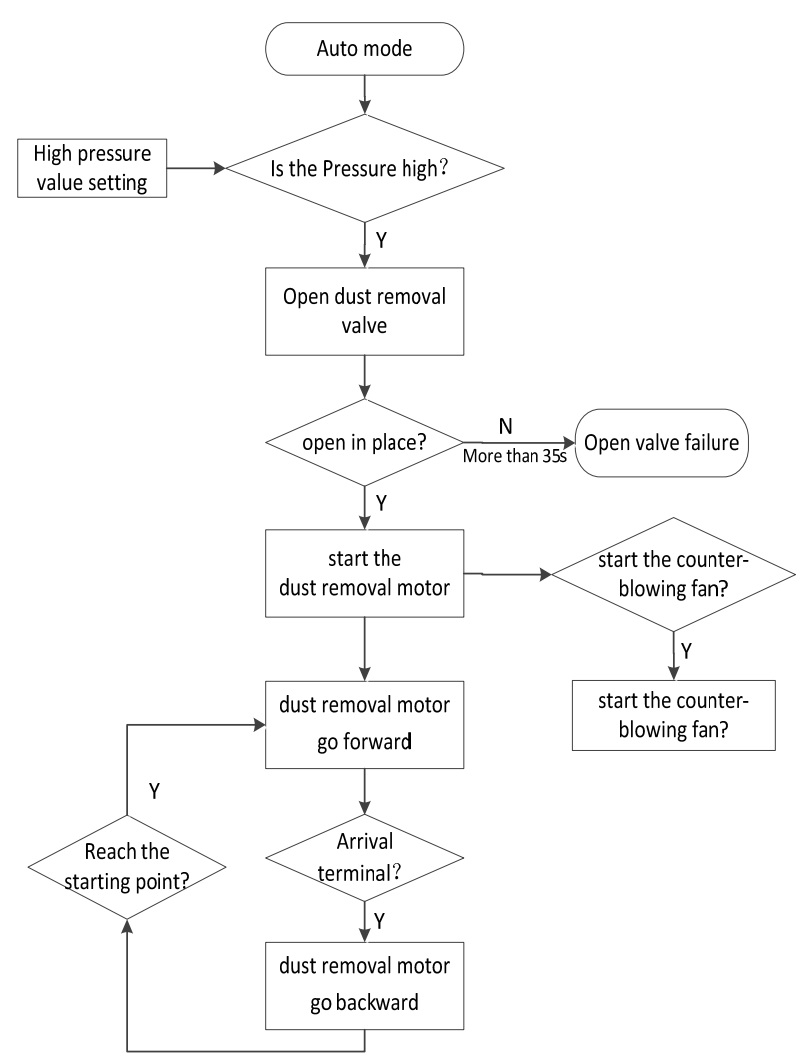

FIGURE I. AUTOMATIC START FLOW CHART OF DUST REMOVAL CONTROL

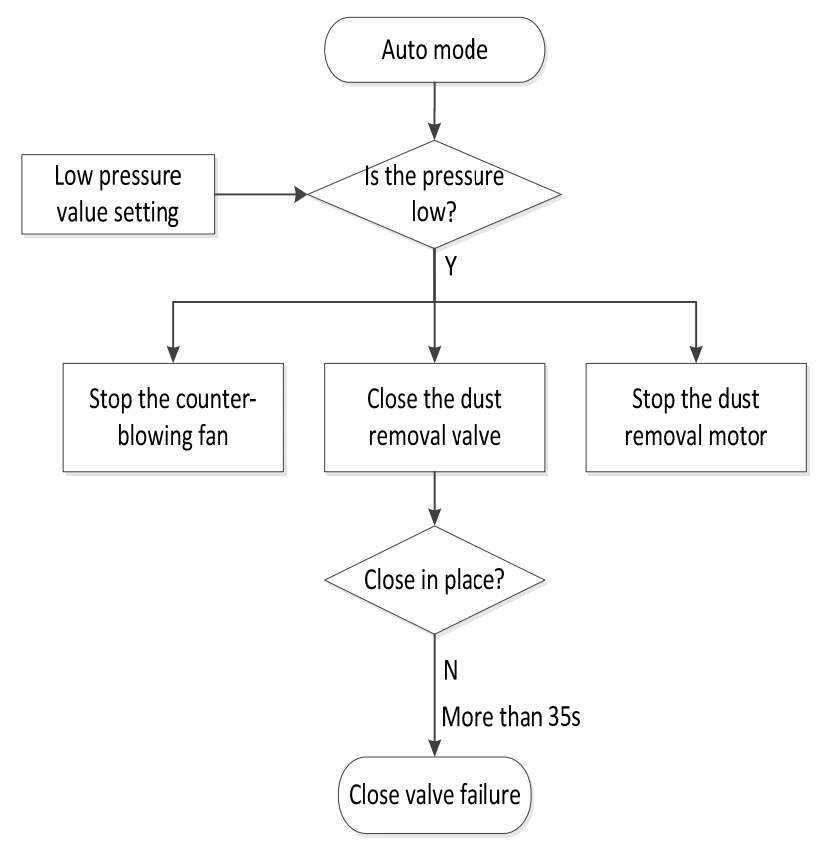

FIGURE II. AUTOMATIC STOP FLOW CHART OF DUST REMOVAL CONTROL
In order to facilitate field personnel monitoring and control dust removal system, Kingview software was used to develop a monitoring picture [3], as shown in Figure III. The field operational personnel can start and stop the dust removal system through the monitoring picture, monitor the operation status of each process equipment in the system, and change the manual/automatic status of the dust removal operation.

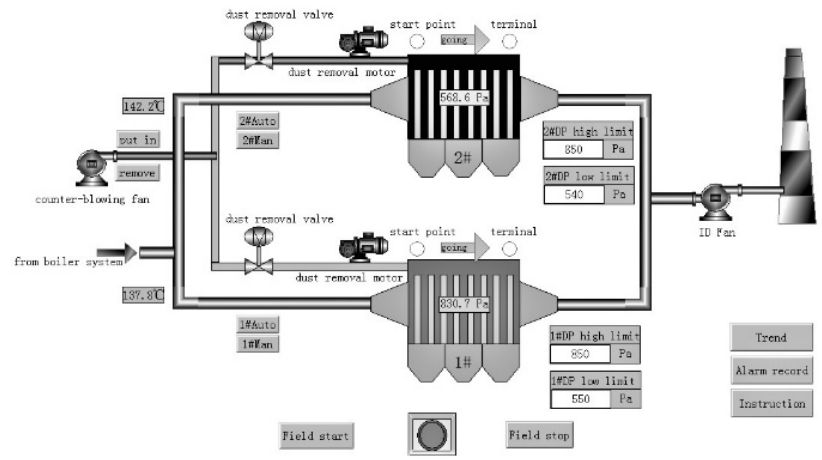

FIGURE III. DUST REMOVAL SYSTEM MONITORING PICTURE

\section{INTEGRATED CONTROL SYSTEM DESIGN}

\section{A. Overall Design}

The plant's main control system uses the Siemens Distributed Control System PCS7 as the controller, the dust removal system uses the Omron PLC as the controller, and the Siemens PCS7 system uses the Profibus-DP fieldbus communication protocol. The Omron PLC uses the DeviceNet fieldbus communication protocol. Therefore, to connect the auxiliary control system to DCS, we must first solve the compatibility problem of heterogeneous protocols. Use inter-network protocol converters (gateways) to achieve compatibility with different fieldbus protocol standards, and build a two-level monitoring system, with PCS7 system as the upper main control system and Omron PLC as the field control subsystem. Realize the integration of main control system and auxiliary control system.

Select the gateway that can achieve Profibus-DP/ DeviceNet protocol data conversion, complete the data format conversion and data read and write operations, and interconnect the DeviceNet protocol network to the Profibus-DP protocol network, to realize the data communication between Siemens PCS7 and Omron PLC hardware devices [4].

DeviceNet and Profibus-DP carry out data transformation through mapping relation, and the gateway PD-100S has Profibus network input buffer and Profibus network output buffer. The data entered by the gateway is stored in the input buffer for Profibus to read. The gateway also extracts data from the network output buffer area and outputs it to the corresponding DeviceNet device by writing commands. PD-100S, as Profibus network node and DeviceNet network node, needs to occupy the location of both network nodes. The principle of data communication is shown in Figure IV. 


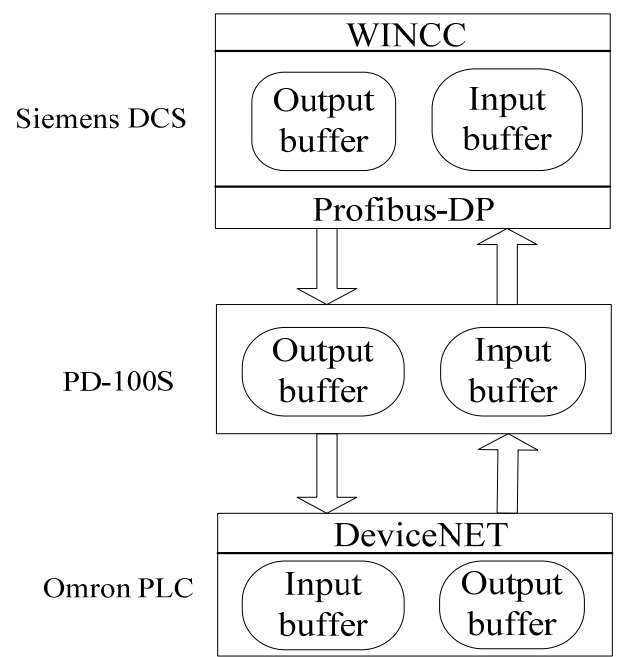

FIGURE IV. PRINCIPLE OF THE DATA COMMUNICATION

Retain the original dust removal monitoring system to facilitate maintenance personnel on-site stop and start dust removal system. The maint control system monitoring picture was developed so that the main control personnel can remotely monitor the dust removal system.

\section{B. System Hardware Structure}

The double-layer supervisory control system includes a main monitoring PC, an main control system PCS7, a field control subsystem Omron PLC, a gateway, a field monitoring PC, field measurement and execution equipments and so on. The system structure is shown in Figure V.

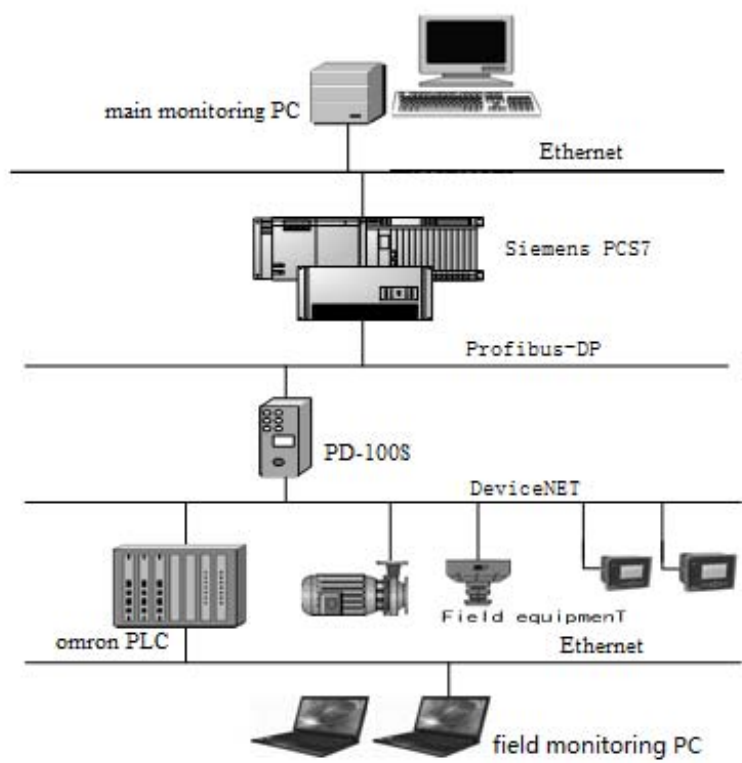

FIGURE V. SYSTEM STRUCTURE

The main control system controller adopts Siemens S7-400 CPU, and the main monitoring PC is a PCS7 server, operator station, engineer station, which can simultaneously complete data collection, information exchange, PCS7 configuration and maintenance[5]. Siemens PCS7 system and main monitoring
PC are connected via Ethernet. The field control subsystem is the Omron PLC system [6], Omron PLC CJ series products are used, and the Omron PLC system and the field monitoring PC are connected via Ethernet [7]. The inter-network protocol converter uses the gateway PD-100S produced by Shanghai Zibo Co., Ltd. The PD-100S supports the interconnection and conversion between the Profibus-DP fieldbus communication protocol and the DeviceNet fieldbus communication protocol. The Siemens PCS7 system is connected to the gateway via the Profibus-DP bus. The Omron PLC system is connected to the gateway via the DeviceNet bus [8] [9].

\section{SYSTEM CONFIGURATION}

\section{A. Siemens PCS7 Configuration}

The Siemens PCS7 system is configured via the main monitoring PC. The configuration process includes: installing the GSD file of the gateway PD-100S under the Siemens PCS7 system [10] [11], hardware configuration of the PCS7 system. Hardware configuration is shown in Figure VI. The hardware configuration includes: creating a target project under the PCS7 system software STEP7 [12]; adding the name and serial number of the actual hardware device of the system under the target project; adding a Profibus-DP network in the PCS7 system, Specify the Profibus-DP master station address, add the address of the gateway PD-100S which is Profibus-DP slave station; Set the address of the gateway PD-100S according to the number of the rotary code switch, and specify the input and output buffer addresses according to the type and the quantity of the remote monitored parameters needed by the dust removal system ; the hardware configuration is downloaded via Ethernet in the Siemens PCS 7 system [13] [14]. The successful download of the hardware configuration indicates that the hardware in the master control system PCS7 has data communication conditions and can interact with the gateway [15][16].

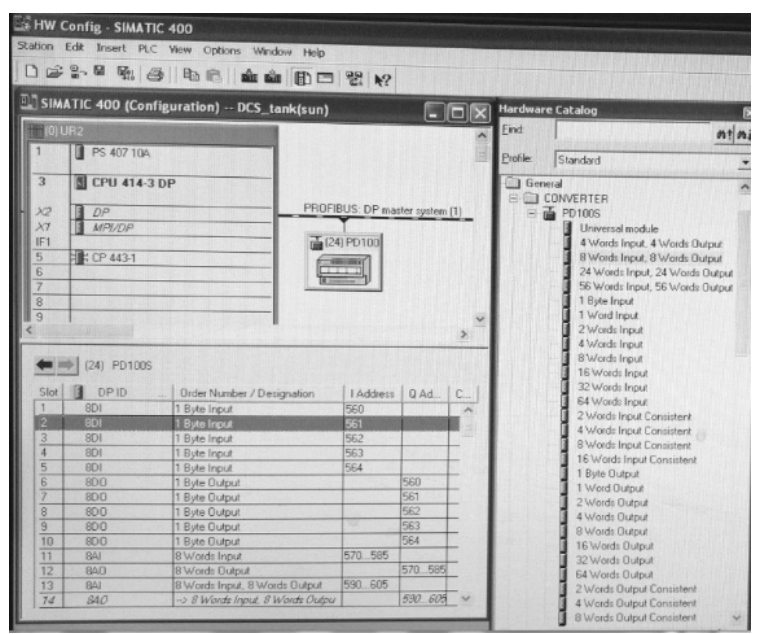

FIGURE VI. HARDWARE CONFIGURATION OF PCS7

\section{B. Gateway PD-100S Configuration}

The gateway PD-100S is the protocol conversion device of DeviceNet to Profibus-DP. It can connect the main station equipment with DeviceNet interface to the Profibus-DP network by setting the relevant parameters, realize protocol 
conversion and data exchange[17]. The PD-100S configuration includes: set input output byte length, network status word, data exchange method and working mode by configuring the super terminal. Set the communication baud rate and station address by setting the rotation code switch and the dial code switch of PD-100S [18].

In this double-layer supervisory system, the gateway PD-100S is used as a slave station of the main control system Profibus-DP. This address is set by the gateway PD-100S rotary code switch. It can be seen from Fig. 6 that the address of PD-100S in PCS7 is 24, that is, the pointer of part A of the rotary code switch on the gateway should rotate to 2 , and the pointer of part B should rotate to 4 . PD-100S is also used as the slave station for the field control subsystem DeviceNet network, and the address is set by the dial code switch of PD-100S.

\section{Omron PLC Configuration}

The Omron PLC system is configured through field monitoring PC. The configuration process includes: installing the EDS file of the gateway PD-100S under the Omron PLC system; hardware configuration of the Omron PLC. The hardware configuration process includes: using $\mathrm{CX}-1$, which is the Omron PLC system software, creates the target project; the scan table is configured under the target project to generate a system identical to the actual hardware system of the Omron PLC system, as shown in Figure VII; generate a DeviceNet network ; generate the modules and address of each station in the DeviceNet network; set the parameters of each module and assign values to the parameters, in order to determine the address of the Omron PLC system I/O variables; determine the IP address of the Ethernet communication module in the Omron PLC system; specify the I/O address used for Omron PLC system to communicate with the PD-100S [19].

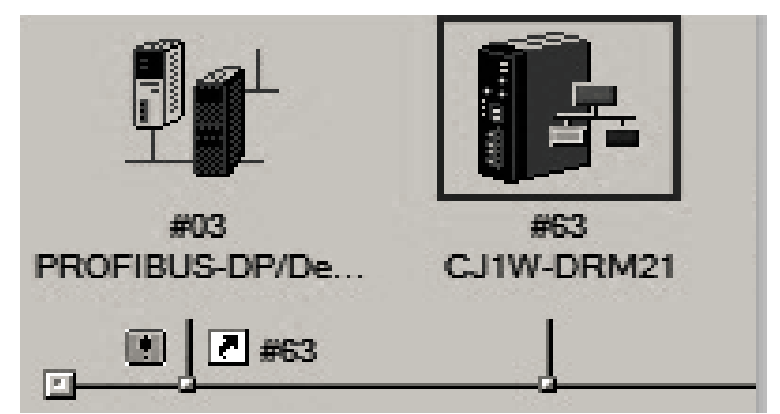

FIGURE VII. THE STRUCTURE OF DEVICENET NETWORK WAS SCANNED AND GENERATED BY THE OMRON PLC SYSTEM

It can be seen from Figure VII that the gateway PD-100S is used as the slave station in the DeviceNet network, and its address is 03 . The address of omron PLC main station module CJ1W-DRM21 is 63.

\section{ENGINEERING STATION CONFIGURATION AND MONITORING PICTURE CREATION OF THE DCS}

On the basis of hardware configuration and successful download of Siemens PCS7, configure the engineering station, create monitoring variables, create a remote monitoring picture [20] in the WINCC software, and use the continuous function chart (CFC) language to group State, establish the connection of the variable and the remote monitoring picture, realize the
PCS7 parameters, equipment status monitoring and remote start-stop control of the field control subsystem [21]. The state of the equipment and its start and stop control information belong to the on-off signal, change the state of the equipment only need to change the variable of the corresponding storage address. The CFC configuration of the starting state and starting control is shown in Figure VIII.

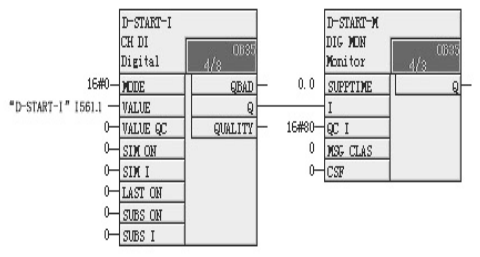

(a) Startup status read back from the field

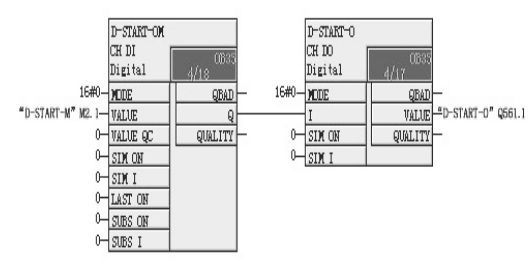

(b) Output of the remote startup command

FIGURE VIII. CFC CONFIGURATION OF REMOTE START-STOP FUNCTION

The temperature of the preheated air and the differential pressure of the dust removal cloth bag are analog value. It needs two bytes of space to store. The CFC configuration of the remote monitoring parameters is shown in Figure IX.

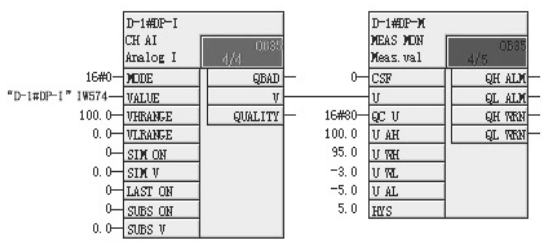

(a) 1 \# differential pressure read back from the field

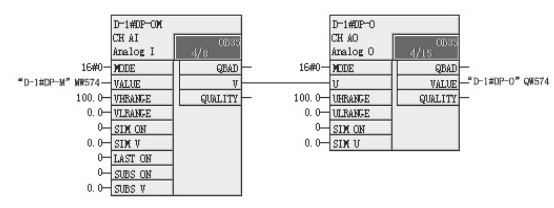

(b) Differential pressure remote setting

FIGURE IX. CFC CONFIGURATION OF REMOTE MONITORING OF FIELD PARAMETERS

The main control system remote monitoring picture is the man-machine interface of the integrated system. Through the remote monitoring picture, the main control operation personnel not only can monitor the start/stop state and operation parometers, but also can control the dust removal system manually, without relying on the field monitoring equipment. The main information of the remote monitoring picture includes: the temperature of the counter-blowing air preheated, the dust removal valve's control status (manual or automatic), the operation status of the dust removal motor, the differential pressure of the dust removal bag, the upper and 
lower limits of the differential pressure setpoint, the start and stop operation soft buttons for centralized control of the remote supervisory control system and so on, as shown in Figure X.

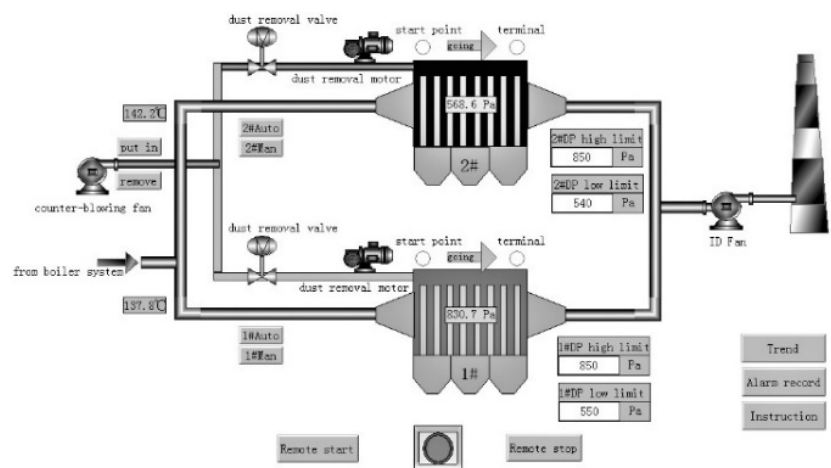

FIGURE X. REMOTE MONITORING PICTURE OF DUST REMOVAL SYSTEM

\section{SYSTEM IMPROVEMENT}

By creating variables and human-machine interface pictures in the Siemens PCS7 system and Omron PLC system, and establishing the connection between the variables and the human-machine interface picture, the field control subsystem can independently control the field process equipment according to the setting control program. And field operators can also monitor the operation parameters and manually control the process equipment through the field monitoring picture of the PC. By constructing the field bus control system based on Siemens DCS and Omron PLC, remote monitoring of field process parameters and equipment status can be realized through the main control system. The centralized control operator also gains higher control authority. They can remote control of field process equipment and actuators, or even start-up or shutdown field system when it required or must.

For the reconstruction and expansion power plant, this heterogeneous fieldbus protocol-compatible double-layer monitoring system can significantly reduce the cost of retrofitting, shorten the construction period, and run stable and reliable under existing data update quantity. In the full use of the DCS control function, it can also get a wider control range and better control quality. The renovation program could be a reference for the plant integration construction.

\section{REFERENCES}

[1] WANG Xiaodong. The application of PLC control system in blast furnace gas dust removal system[J]. Automation application, 2016, (5): 54-55.

[2] ZHANG Dianyin, WANG Haitao. Dust removal equipment and operation management[M]. Beijing: Metallurgical industry press, 2010; 25-35

[3] YANG Xianhui. Fieldbus technology and its application[M]. Beijing: Tsinghua university press, 2008: 56-60.

[4] XIAO Sheng, WANG Chunli. Appliation of fieldbus technology in control system of units[J]. Thermal power generation, 2011, 40(2): 69-71.

[5] YIN Jiang, FENG Jiangtao. Power plant decentralized control system[M]. Beijing: China electric power press, 2006: 7-12.

[6] YANG Jinping, SUN Zhumei. Principle and application of programmable controller[M]. Beijing: China electric power press, 2013: $35-47$.
[7] SHEN Rongjuan. Design and implementation of a Web based B/S mode multi-field bus teaching experiment platform[D]. Nanjing: Southeast university, 2015: 22-26.

[8] TAN Xiangshuai, CHEN Kun, GAO Kui. Fieldbus commissioning method and problem handling for coal-fired power plants[J]. Thermal power generation, 2016, 45(5): 117-120.

[9] WANG Qi, CHENG Chen, SUN Zhumei, et al. Communication compatible design of Profibus-DP and Modbus protocol[J]. Industrial Instrumentation \& Automation, 2016, (06): 53-56+83.

[10] SUN Zhumei, PING Guonan, WANG Qi. Heterogeneous integration of Siemens DCS and Modbus PLC[J]. Automation \& Instrumentation, 2016, (12): 47-49+52.

[11] ZHANG Shuai, SHA Ting, NAN Hongwei. Design of PLC and DCS communication system based on Profibus-DP protocol[J]. Technology information, 2016, 14 (25): 16-17.

[12] FU Weiming, CHEN Weifang, MA Wantai. Interface design of ac servo driver based on DeviceNet protocol[J]. Industrial control computer, 2012, 25(11): 16-17+20.

[13] CHEN Jing. Application of Profibus fieldbus technology in new power plant[D]. Beijing: North China electric power university, 2016: 48-52.

[14] CAO Wei. Research on the control system of inverter based on PLC[J]. Digital technology and application, 2014, (05): 23.

[15] HAN Jian, CHANG Weiwu, LI Yadu, et al. The application of FF and Profibus-DP bus in power plant auxiliary network system[J]. Chemical automation and instrumentation, 2016, 44(2): 215-217.

[16] HAN Chao, JIA Jing. Diagnosis and analysis of application faults of profibus fieldbus technology in thermal power plants[J]. Electric power, 2016, 49(2): 6-9.

[17] DeviceNet/ Profibus-DP converter pd-100s product manual. Shanghai sibo automation technology co. LTD.

[18] PING Guonan, WANG Qi, GUAN Yanpeng, et al. Communication of multi - field bus control system based on Siemens PCS7[J]. Automation and Instrumentation, 2018, 33(2): 82-85.

[19] TANG Zhongyan. Application of network control system based on omron PLC[J]. Electrician technology, 2011, (11): 41-43.

[20] SUN Zhumei, YANG Jinping LAI, Changsheng. PLC experimental training course[M]. Beijing: China electric power press, 2009: 110-125.

[21] LI Weimin. Application of Profibus field bus control system in thermal power plant[J]. Automation application, 2016, (5): 83-84. 